\title{
LA CARTA DE LEIBNIZ A MAGNUS WEDDERKOPF: EL ESQUEMA NECESITARISTA DE 1671
}

\author{
Maximiliano Escobar Viré \\ Universidad de Buenos Aires - Universidad Nacional \\ de Quilmes - ex becario CONICET \\ m_escobarvire@yahoo.com
}

\begin{abstract}
RESUMEN / ABSTRACT
En una conocida carta redactada en 1671, Leibniz formula un esquema argumental que intenta resolver el problema de la existencia del mal, pero que sorprende por una conclusión necesitarista, según la cual toda la cadena de eventos existentes es la óptima, y por tanto es necesaria. El presente trabajo propone una reconstrucción detallada del argumento y un análisis de su significación modal. Este análisis revela que la necesidad atribuida por Leibniz al mundo actual expresa la fuerza modal que adquiere lo óptimo en su filosofía, ya en 1671, como principio que rige indefectiblemente la acción de Dios.

Palabras Clave: necesitarismo, óptimo, necesidad absoluta, principio de razón suficiente, libertad.

\section{LEIBNIZ'S LETTER TO MAGNUS WEDDERKOPF: THE NECESSITARIAN OUTLINE OF 1671}

In a well-known letter written in 1671, Leibniz draws an argumentative outline which tries to resolve the problem of evil, but that also astonish us with a necessitarian conclusion, according to which the whole chain of the actual events is the best, and therefor is necessary. The present work proposes a detailed reconstruction of the argument and an analysis of its modal meaning. This analysis shows that the necessity assigned by Leibniz to the actual world expresses the modal force that the best acquires in his philosophy, even in 1671, as the principle that rules unavoidably every action of God.
\end{abstract}

KEYWORDS: necessitarianism, the best, absolute necessity, principle of sufficient reason, freedom.

\section{Introducción}

R⿴囗十 En el proceso de elaboración de la filosofía de Leibniz, hay al menos dos momentos o períodos en los que se puede atribuir al filósofo (con bastante consenso entre los comentadores) una concepción metafísica que cabe caracterizar como necesitarista. El primero de tales momentos alcanza su formulación más clara en la carta que Leibniz envió en mayo de 1671 al entonces profesor de jurisprudencia de la Universidad de Kiel, Magnus Wedderkopf. En dicha carta, Leibniz sostiene sin más la tesis según la cual "todo lo que ha sucedido, sucede o sucederá [...] es necesario" (Leibniz, A II, i, 117). El segundo período se encuentra representado por algunos de 
los numerosos escritos metafísicos que Leibniz redactara durante su estancia en París, en 1676, y que se hallan reunidos en la sección titulada como De summa rerum en la edición de la Academia ${ }^{1}$.

Solo en este segundo momento puede encontrarse en Leibniz una vivaz influencia del pensamiento de Spinoza ${ }^{2}$, la cual no parece haber sido decisiva en el tema específico de la necesidad con la cual se siguen los seres finitos (o modos) a partir de la naturaleza divina $^{3}$. En cambio, el necesitarismo de 1671 es de propia facturación leibniziana, como lo revela su apelación al principio de razón suficiente. Más aun, el esquema argumental de 1671 contiene numerosos elementos teóricos que habrían de perdurar en la metafísica del filósofo de Hannover, aun cuando el contenido necesitarista fuese ulteriormente desterrado de su obra. El presente trabajo se propone reconstruir este esquema argumental y analizar su significación modal, precisamente a la luz de las ideas modales que la evidencia textual permite detectar en el Leibniz de 1671. Se intentará precisar en qué sentido, o bajo qué cualificación, cabe emplear el término "necesitarismo" para calificar al esquema en cuestión. Este análisis permitirá, asimismo, evaluar la importancia de este primer esbozo de una explicación metafísica de la existencia del mundo actual, en relación con el posterior desarrollo de la filosofía leibniziana.

\section{Reconstrucción del esquema argumental}

La famosa carta a Magnus Wedderkopf constituye uno de los documentos más tempranos que nos han llegado en los que Leibniz tematiza, con cierto detenimiento, la problemática del mal ${ }^{4}$. Es en tal contexto que Leibniz propone un esquema argumental pretencioso, en un pasaje que conviene citar in extenso:

1 Cf. A VI, iii, 461-588. Sobre la orientación necesitarista de la metafísica leibniziana hacia el final del período de París, cf. Rescher 1981, pp. 106-108.

2 En efecto, no hay indicios fiables de que Leibniz haya tenido algún acceso a la metafísica de Spinoza antes de su rencuentro con Tschirnhaus en París, en 1675. Esto parece contrariar su propio testimonio, expuesto en los Nuevos ensayos sobre el entendimiento humano. Allí Leibniz declara haber comenzado a pensar del lado de los spinozistas, "que no dejan más que una potencia infinita en Dios, sin reconocer ni perfección ni sabiduría en relación a él”, lo cual deriva en "una necesidad bruta" (cf. A VI, vi, 73). Por esta razón, la relación Spinoza-Leibniz no será abordada en este trabajo. Acerca de la influencia del pensador holandés sobre el alemán antes de 1675, cf. Laerke 2008, pp. 361-373; también 553-556.

3 Así puede inferirse a partir de las notas que Leibniz redacta, entre octubre de 1675 y febrero de 1676, sobre las ideas de Spinoza que le comunica Tschirnhaus. En ellas, la tesis necesitarista no se encuentra, y apenas puede inferirse a partir de la afirmación según la cual "solamente Dios es libre". Cf. A VI, iii, 384-385.

4 Por cierto, el primer "documento" sigue siendo la anotación al Compendium metaphysicum de Daniel Stahl, de 1663-1664 (cf. A VI, i, 27). De 1668-1669 datan algunos pasajes breves, en los que Leibniz enuncia algunas ideas sobre el carácter accidental de la existencia del mal (cf. A VI, i, 496 c.18). Hay que esperar hasta 1670-1671 para encontrar un escrito más 
[...] es necesario que todo sea resuelto en alguna razón, y no parar hasta llegar a la primera razón; de otro modo debería admitirse que algo puede existir sin una razón suficiente de su existencia, admisión que destruye la demostración de la existencia de Dios y de muchos teoremas filosóficos. ¿Cuál es entonces la última razón de la voluntad divina? El intelecto divino. Porque Dios quiere aquellas cosas que él entiende que son las mejores y las más armoniosas, y las elige, como si fuera, a partir de un número infinito de todos los posibles. ¿Cuál es entonces [la razón última] del intelecto divino? La armonía de las cosas. ¿Y cuál es la de la armonía de las cosas? Nada. Por ejemplo, por qué la proporción entre 2 y 4 es también la que se da entre 4 y 8, de ello no puede darse ninguna razón, ni siquiera por la voluntad divina. Eso depende de la misma esencia o idea de las cosas. Por tanto, las esencias de las cosas son como los números, y contienen la posibilidad misma de los entes; Dios no produce tal posibilidad, produce la existencia; o más bien, porque esas mismas posibilidades o esencias de las cosas coinciden con Dios mismo. Sin embargo, como Dios es la mente más perfecta, es imposible que él mismo no sea afectado por la armonía más perfecta, y por tanto, también lo es que no sea necesitado hacia lo óptimo por la misma idealidad de las cosas. Lo cual en nada disminuye la libertad. Puesto que la suprema libertad es ser compelido a lo que es óptimo por la recta razón; quien desea otra libertad es necio. De aquí se sigue que todo lo que ha sucedido, sucede o sucederá es lo óptimo, y por tanto es necesario, pero como dije, con una necesidad que nada quita a la libertad, puesto que nada quita a la voluntad y al uso de la razón (Leibniz, A II, i, 117).

El esquema argumental contenido en este pasaje puede reconstruirse, a mi juicio, como un encadenamiento de cuatro argumentos.

El primero de tales argumentos puede formularse del siguiente modo:

1. Es necesario que todo sea resuelto en alguna razón suficiente.

2. Es necesario que toda razón suficiente sea resuelta en otra, hasta alcanzar una razón primera de todas las cosas.

3. Por tanto, es necesario que todo sea resuelto en la razón primera de todas las cosas.

Este primer argumento muestra ya la importancia que posee el principio de razón suficiente en la metafísica que Leibniz se propone construir. Tal principio se encontraba ya enunciado en la Demonstrationum catholicarum conspectus de 1668-1669, como la primera vía propuesta por Leibniz para la demostración de la existencia de Dios 5 . Asimismo, en un escrito de la misma época de la carta a Wedderkopf, la Demonstratio

extenso que aborde la cuestión, como será el Von der Allmacht und Allwissenheit Gottes und der Freiheit des Menschen (A VI, i, 537 y ss.).

$5 \quad$ Cf. A VI, i, 494 c. 1. 
propositionum primarum ${ }^{6}$, Leibniz ofrece una demostración del propio principio, y aclara un poco más qué entiende por "razón suficiente".

En la física escolástica, como bien señala Carraud, la expresión "ratio sufficiens" se había vuelto común para nombrar la causa que es suficiente, por sí, para producir el efecto7. Se empleaban también expresiones como "causa sufficiens" o "causa plena", para aludir a la combinación de causas parciales que, una vez dada, volvía necesaria (en el sentido de una necesidad natural) la producción del efecto. También se hablaba de "causas requeridas" o "requisitos" para referir a cada una de las causas coadyuvantes tomadas aisladamente ${ }^{8}$. Leibniz abreva en esta tradición, aunque extiende la jurisdicción del principio a todos los eventos, incluidos los actos libres 9 . Como puede verse ya en la Confessio naturae contra atheistas de 1668, Leibniz reclama la "ratio plena" de los fenómenos físicos ${ }^{10}$, y encuentra que dicha ratio no puede nunca inferirse de las solas cualidades geométricas. En la Demonstratio propositionum primarum, define a la "ratio sufficiens" como aquella que, "si es dada, la cosa es" (Leibniz, A VI, ii, 483). A su vez, dicha ratio se identifica con la totalidad de los requisitos de una cosa: "todos los requisitos son la razón suficiente [de la cosa]" (Leibniz, A VI, ii, 483). Y un requisitum es aquello que, "si no es dado, la cosa no es" (Leibniz, A VI, ii, 483).

Así, la primera premisa del primer argumento puede interpretarse como afirmando que, para todo lo que existe, necesariamente debe haber una razón que permita explicar todos y cada uno de los aspectos de aquello que existe. En otras palabras, es necesario que haya algo que contenga todos los requisitos que son necesarios para que la cosa exista y sea del modo en que es, tal como Leibniz lo aclara en Von der Allmacht und Allwissenheit Gottes und der Freiheit des Menschen ${ }^{11}$. Aquí aparecen formulados los dos ejes en los que el principio de razón suficiente debe dar razón: el de la existencia y el de la esencia de las cosas existentes. La resolución a la que Leibniz refiere alude al procedimiento lógico de descomposición de una noción compleja en las notas del definiens. Ello muestra el carácter más bien lógico que Leibniz parece trasladar a toda relación causal. Como señala Francesco Piro, Leibniz extiende un modelo lógico de explicación (empleado por los escolásticos en el terreno de la física) a todos los eventos, lo cual implica la asimilación de los requisitos en general con condiciones lógicas, y no solo fácticas. Esto podría haberlo motivado, según Piro, a emplear el término "razón" en lugar del de "causa"12.

El segundo argumento constituye una explicitación del primero, en la medida en que i) enuncia cuál es la razón suficiente de la existencia de todas las cosas y

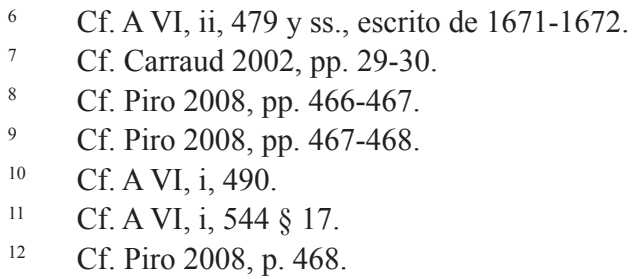


ii) muestra de qué modo esa razón se resuelve en la razón primera. Su reconstrucción sería la siguiente:

1. La razón última de la voluntad divina es el intelecto divino.

2. La razón última del intelecto divino es la armonía de las cosas, la cual abarca todo aquello que "depende de la misma esencia o idea de las cosas".

3. Las esencias de las cosas son independientes de la voluntad divina, contienen la posibilidad de las cosas y "coinciden con Dios mismo".

4. No hay ninguna razón para la armonía de las cosas (o más bien, no hay ninguna razón fuera de ella misma).

5. Por tanto, la razón suficiente última de todas las cosas es la armonía de las cosas (la cual parece coincidir con Dios). [Conclusión implícita]

En la interpretación que propongo, este segundo razonamiento presupone una premisa implícita, según la cual

La razón suficiente de la existencia de las cosas actuales es la voluntad divina.

En efecto, Leibniz está especificando de qué modo es que todo se resuelve en la razón primera. Y para ello comienza preguntando cuál es la ratio ultima de la voluntad divina, lo cual presupone que es dicha voluntad la que media entre el entendimiento divino y la existencia de las cosas.

Este argumento condensa, sin desarrollar, mucha metafísica. No se aclara qué es la harmonia rerum, ni en qué sentido se afirma la identificación de las esencias con Dios mismo. Algo de esta metafísica puede reponerse a partir de los escritos leibnizianos de los ocho años que preceden a la carta aquí abordada. Leibniz parece haber tomado la concepción de una armonía universal a partir de la obra de Johann Heinrich Bisterfeld, como puede verse en las anotaciones que hizo referidas a obras de este autor hacia 1663-1666 ${ }^{13}$. Allí se presenta dicha armonía como un "nexo simbiótico", una "unión y comunión" entre todas las cosas, que surge del hecho de que ningún ente (con excepción de Dios) puede ser "absoluto", por lo cual no puede existir en soledad ${ }^{14}$. Esta conexión debe tener lugar primero en el plano de las esencias, dado que "la belleza de las cosas" constituye, en la Confessio naturae contra atheistas, la razón por la cual Dios elige estas $\operatorname{cosas}^{15}$. Hacia 1668-1669, Leibniz afirma que la intuición "cara a cara" de Dios (la visión beatífica) es la contemplación de "la armonía universal de las cosas", "porque Dios no es nada distinto a la armonía de las cosas o al principio de la belleza en ellas mismas"

13 Cf. A VI, i, 151-161. Sobre la concepción de Bisterfeld de la armonía universal y su influencia sobre Leibniz, cf. Antognazza 1999.

14 Cf. A VI, i, 153.

15 Cf. A VI, i, 492. Leibniz ratificará esta idea en la Confessio philosophi de 1672-1673. Cf. A VI, iii, 146. 
(Leibniz, A VI, i, 499). Y en los Elementa juris naturalis, hacia 1671, Leibniz define ya la armonía como la "diversidad compensada por la identidad" (Leibniz, A VI, i, 484).

Parece entonces que inquirir por la razón de la harmonia rerum sería tanto como buscar la razón por la cual las esencias son como son, lo cual equivale a buscar la razón de Dios mismo (o de su entendimiento). El Leibniz de 1671 no parece sentir la necesidad de brindar tal razón, como sí la sentirá en 1676, cuando encare la revisión del argumento ontológico.

Hasta aquí, sin embargo, el argumento parece quedar incompleto. Se afirma que la razón suficiente última de todo reside en la armonía de las cosas, pero no se explica de qué modo surgen las cosas existentes a partir de tal principio. El tercer argumento viene a especificar esta cuestión. Él mismo puede formularse citando al propio Leibniz:

1. como Dios es la mente más perfecta, es imposible que él mismo no sea afectado por la armonía más perfecta, y por tanto, también lo es que no sea necesitado hacia lo óptimo por la misma idealidad de las cosas.

2. Por tanto, "todo lo que ha sucedido, sucede o sucederá es lo óptimo".

3. En consecuencia, "todo lo que ha sucedido, sucede o sucederá [...] es necesario".

Aquí se encuentra el foco central del interés de este trabajo: la conclusión modal. Leibniz infiere que todos y cada uno de los eventos del mundo actual son necesarios. También aclara que la existencia necesaria de todo lo actual se sigue del hecho de que Dios, siendo la mente más perfecta, es "necesitado hacia lo óptimo". Si se recuerda la premisa implícita del segundo argumento, debe interpretarse que es la voluntad divina la que es conducida necesariamente a querer o elegir lo más perfecto. Tal es, a mi juicio, el corazón del necesitarismo leibniziano de 1671 (si cabe denominarlo así).

Finalmente, el cuarto argumento no especifica a los anteriores, sino que se propone salvar la libertad de Dios (aportando lo que puede considerarse como el contenido compatibilista del esquema argumental). Su reconstrucción podría formularse como sigue:

1. La suprema libertad consiste en que la voluntad sea compelida a elegir lo que el entendimiento juzga como lo mejor.

2. La voluntad de Dios es necesitada a elegir lo que su entendimiento juzga óptimo.

3. Por tanto, Dios actúa libremente.

4. Por tanto, el carácter necesario de todos los eventos no suprime la libertad de Dios.

Como puede verse, la pretensión del Leibniz de 1671 es conciliar la necesidad de los eventos con la libertad de Dios. En ello cabe apreciar cuán lejos se halla la orientación de su metafísica juvenil respecto del necesitarismo spinozista. 


\section{El problema de la significación modal del esquema leibniziano de 1671}

Muchos son los interrogantes que este esquema argumental deja abiertos. El que interesa abordar aquí se refiere al esclarecimiento de la significación modal que cabe atribuir a la conclusión del tercer argumento formulado en la presente reconstrucción.

En efecto, en el ámbito de la filosofía escolástica de los siglos XVI y XVII (tanto en sus vertientes católicas como protestantes), las nociones modales en general, y la noción de necesidad en particular, eran objeto de numerosos análisis, que redundaban en diversas distinciones y clasificaciones ${ }^{16}$. Tales precisiones conceptuales resultaban imprescindibles para el abordaje de los problemas filosóficos y teológicos que se venían discutiendo con especial intensidad desde la Reforma: la predestinación, la libertad, el decreto de salvación y de condenación, etc. Era común, por ejemplo, diferenciar la necesidad metafísica (referida a lo que no puede ser de otro modo por la propia naturaleza esencial de las cosas) respecto de la necesidad fisica (fundada en ciertas leyes naturales cuya vigencia podría ser suspendida por milagro). Más aun, como señala Sven Knebel, podía encontrarse también en los autores escolásticos una necesidad moral, noción que cualificaba los actos libres de los espíritus y que entrañaba un tercer orden de modalidades (aquel de las modalidades morales) ${ }^{17}$. Otra distinción importante en el abordaje de los problemas mencionados era aquella, planteada por Pedro Abelardo, entre una necesidad in sensu composito (luego caracterizada como de dicto) y otra in sensu diviso (o de re) ${ }^{18}$. Pero la distinción escolástica que más parece haber influido sobre Leibniz era aquella entre una necesidad absoluta y una necesidad hipotética. El filósofo de Hannover incorporó esta distinción como un componente estable y fundamental de su metafísica. Siguiendo el ejemplo de Tomás de Aquino, Leibniz asoció la necesidad absoluta con la necesidad del consecuente, por un lado, y la necesidad hipotética con la necesidad de la consecuencia, por otro. Esta última modalidad se predicaba sobre una proposición condicional, y constituía la versión escolar de la tradicional necesidad condicional boeciana ${ }^{19}$.

Cabe entonces plantear el siguiente interrogante: ¿cuál es el tipo de necesidad que Leibniz está atribuyendo a todos los eventos en la carta a Wedderkopf? ¿Se trata de una necesidad absoluta o hipotética? ¿Se trata acaso de una necesidad que no pertenece a estas dos categorías?

16 Sobre la importancia de las distinciones escolásticas en el contexto de formación de Leibniz, cf. Schepers 1999 y Grua 1953, p. 127.

17 Cf. Knebel 2003. Por supuesto, Leibniz conocía la noción de necesidad moral desde joven, y la empleó de modo persistente en su metafísica de la época de los Ensayos de Teodicea.

18 Sobre la formulación abelardiana de esta distinción, cf. Knuuttila 2008, pp. 520-521, 533535.

19 Cf. G III, 400; G VI, 380. La asociación tomasiana entre la necessitas absoluta y la necessitas consequentis, así como entre la necessitas consequentiae y la necesidad condicional boeciana, se encuentra en Quaestiones disputatae de veritate, q. 24, art. 1, ad 13. 


\subsection{La interpretación en términos de necesidad absoluta}

El concepto de necesidad absoluta que Leibniz recibe en su formación e incorpora en su metafísica era explicado en términos lógicos: algo es absolutamente necesario cuando su contrario o su negación implica contradicción. Así se lo encuentra, por ejemplo, en el Compendium metaphysicum de Daniel Stahl, texto que Leibniz leyó y anotó en 1663-1664 ${ }^{20}$. Allí, en la tabula XII (que Leibniz deja sin comentar), Stahl distingue la necesidad absoluta respecto de la necesidad hipotética, y define lo absolutamente necesario como aquello "cuyo opuesto implica contradicción"21. A esta caracterización lógica, Leibniz le asocia tempranamente una caracterización epistémica de las nociones modales en general. Ya en el texto de la Confessio philosophi de 1672-1673, Leibniz propone denominar como "necesario" "a aquello cuyo opuesto implica contradicción, es decir, lo que no se puede entender claramente" 22 . Y en las notas de su conversación con Niels Stensen de fines de 1677, Leibniz introduce por fin la distinción entre esta necesidad absoluta o per se y la necesidad hipotética o per accidens:

Necesidad absoluta es cuando la cosa no puede ser pensada de otra manera, sino que esto implica una contradicción en los términos; v.g., que tres por tres sean diez.

Necesidad hipotética es cuando la cosa puede ser pensada de otro modo por sí misma [per se], pero es necesaria por accidente [per accidens], en razón de estar presupuestas otras cosas exteriores a ella misma; v.g., era necesario que Judas fuese pecador, suponiendo que Dios lo hubiese previsto. O bien suponiendo que Judas lo hubiese considerado como óptimo (Leibniz, A VI, iv, 1377).

La temprana lectura del manual de Stahl es prueba suficiente de que el Leibniz de 1671 de ningún modo podía desconocer la distinción entre las dos clases de necesidad que luego adoptaría ${ }^{23}$.

Hay buenas razones para interpretar que la necesidad atribuida a todos los eventos en la carta a Wedderkopf es la necesidad absoluta. La primera de tales razones es el testimonio posterior del propio Leibniz, que parece avalar tal interpretación. En un conocido pasaje de un escrito de 1689, titulado por la edición de la Academia como De libertate, contingentia et serie causarum, providentia, Leibniz comienza confesando

Cf. A VI, i, 21 y ss.

Stahl, p. 73.

De Olaso 112 / A VI, iii, 126.

23 En una nota sobre la Philosophia practica de Jacob Thomasius, también de 1663-1664, Leibniz incluso emplea la distinción en un breve y desconcertante argumento, en el cual parece afirmar que Dios no estaría obligado de modo inmutable a cumplir las leyes del derecho natural. Cf. A VI, i, 52. Para un análisis de este argumento, cf. Rateau 2008, pp. 64-66. El Leibniz posterior habría de pasar más bien a la posición contraria, como lo sugiere su ubicación de la doctrina del derecho entre las ciencias referidas a verdades necesarias o eternas. 
cuán cerca estuvo en su juventud respecto de la consideración de todas las cosas como "absolutamente necesarias":

Yo, cuando había considerado que nada sucede por casualidad, ni por accidente [...] y que la fortuna separada del destino es una palabra vacía, y que nada existe si no se dan [sus] requisitos singulares -si bien de todos ellos se sigue lo mismo, el que la cosa exista-, poco me había alejado [entonces] de la opinión de aquellos que consideran todas las cosas absolutamente necesarias, y que juzgan que es suficiente estar exento de coacción para que haya libertad, aunque se esté sometido a la necesidad [...] (Leibniz, A VI, iv, 1653).

Asimismo, en la copia que Leibniz conservó de la carta a Wedderkopf, posteriormente anotó:

Luego corregí esto. Porque una cosa es que los pecados vayan a ocurrir infaliblemente, y otra cosa es que vayan a ocurrir de modo necesario (Leibniz, A II, i, 118).

Esta anotación sugiere que la corrección realizada sobre el contenido de la misiva consistió en diferenciar dos categorías que su metafísica de madurez se cuidaría de no confundir: las de lo infalible y lo necesario. El error de 1671 habría sido, pues, el de extender, a todo lo que acontece de modo infalible, la categoría de necesidad en su sentido primario y fundamental: la necesidad absoluta.

Otra razón que puede esgrimirse tiene que ver con el empleo de las nociones modales que hace Leibniz en otro escrito importante del mismo período referido al problema de conciliar la perfección divina con la existencia de los pecados: el Von der Allmacht und Allwissenheit Gottes und der Freiheit des Menschen. Allí, Leibniz intenta operar una clarificación del concepto de posibilidad como vía argumental para responder al sofisma que pretende inferir la necesidad de la condenación a partir de la presciencia divina $^{24}$. En tal contexto, Leibniz escribe:

[...] aquello que se enmaraña y autocontradice es tenido por imposible; [...] Así pues, es posible aquello que resulta susceptible de ser explicado con claridad y sin confusión ni autocontradicción alguna (Leibniz, Roldán 64 / A VI, i, 540).

En este pasaje puede verse nuevamente la tendencia a caracterizar las nociones de posible y de imposible en términos lógicos de consistencia-contradictoriedad, tanto como en términos epistémicos de concebibilidad clara. Es fácil inferir que la delimitación conceptual de las restantes modalidades debiera seguir la misma vía de explicación, y que la noción de necesidad resultante debiera ser la de necesidad absoluta: aquello cuyo contrario se contradice y no se puede entender de modo claro. En consecuencia, habría que reconocer que, cuando el Leibniz de 1671 emplea la categoría de "necesidad" (incluso en el contexto teológico y metafísico del problema que oponía la predestinación 
a la libertad y la contingencia), la noción modal que tiene en mente no es otra que la tradicional necesidad absoluta, que años más tarde sería introducida definitivamente en el cuadro conceptual de su metafísica.

2.2. En contra de la interpretación de la necesidad del esquema como necessitas absoluta

La interpretación hasta aquí bosquejada enfrenta dificultades que, a mi juicio, resultan prácticamente insalvables. La primera y principal dificultad consiste en reconocer que, en la carta a Wedderkopf, Leibniz hace un uso del concepto de necesidad que luego rechazará y considerará incompatible, precisamente, con la noción de necessitas absoluta.

En efecto, si se atiende al tercer argumento de la reconstrucción aquí formulada, resulta imprescindible notar que la necesidad que se atribuye a la totalidad de los eventos es una consecuencia del hecho de que i) tales eventos constituyen "lo óptimo" y ii) Dios es "necesitado hacia lo óptimo" por la armonía más perfecta. En otras palabras, la necesidad de los eventos es una necesidad derivada, que se infiere como consecuencia de dos condiciones, una de las cuales (la tendencia de la voluntad divina hacia lo óptimo) es claramente exterior a la naturaleza o esencia de las cosas creadas. Pero esto significa que dicha necesidad no constituye una necesidad per se, no surge de la propia esencia de las cosas; estas podrían ser pensadas de un modo diferente sin implicar contradicción alguna. Y ello significa (siguiendo la caracterización que Leibniz formulará en las notas del diálogo con Stensen) que la necesidad atribuida a la serie de cosas existentes no puede ser una necesidad absoluta.

El Leibniz de la carta a Wedderkopf parece estar admitiendo, sin más, un principio lógico-modal según el cual la consecuencia necesaria de una verdad necesaria es, también, una verdad necesaria ${ }^{25}$. Este principio es aceptado actualmente como uno de los principios básicos de la lógica modal proposicional: el denominado Axioma de distribución. El mismo suele formularse del siguiente modo:

$$
\square(A \rightarrow B) \rightarrow(\square A \rightarrow \square B)^{26}
$$

En el esquema de la carta, la posición de Leibniz parece adecuarse parcialmente a este axioma: como es necesario que Dios sea compelido hacia lo óptimo, es necesario que pase a la existencia la cadena de eventos que es la más perfecta (“a partir de un número infinito de todos los posibles"). A partir de esta aplicación del axioma, Leibniz da un paso más, que puede ser calificado como inválido: infiere la necesidad de la existencia de este mundo (esta cadena de eventos) a partir de la necesidad de la existencia de lo

25 Varios comentadores han notado esto. Cf. por ejemplo S1 xxv; Adams 1994, p. 16.

26 Cf. Garson 2006, p. 30 y 2009. Como señala Garson, este axioma constituye uno de los dos principios (junto a la Regla de necesitación) que forman el denominado "sistema K". En esta formulación, las letras "A" y "B" constituyen meta-variables proposicionales. 
óptimo en general. Aquí puede objetarse que la aplicación del Axioma de distribución resulta incorrecta hasta tanto no se demuestre que este mundo es necesariamente el óptimo. De hecho, esto es lo que el propio Leibniz objetará en sus años de madurez, cuando afirme que es contingente el que este mundo sea el óptimo ${ }^{27}$.

Aun así, lo que interesa notar es que la necesidad que se atribuye a los eventos creados en la carta de 1671 no es la necesidad absoluta o per se, propia de aquello cuya esencia contraria conlleva contradicción. Por el contrario, se trata de la necesidad que hace que Dios quiera lo óptimo, en razón de ser la mente más perfecta: "todo lo que ha sucedido, sucede o sucederá es lo óptimo, y por tanto es necesario". Se trata, pues, de una necesidad transitiva, que se transfiere del antecedente al consecuente.

Una de las primeras tareas que Leibniz emprenderá, ya en 1672, será la de rechazar esta concepción de la necesidad, basada en el denominado Axioma de distribución. Leibniz pasará a sostener que algo puede ser consecuencia necesaria de una verdad necesaria sin ser, por ello, algo en sí mismo necesario. En la Confessio philosophi de 1672-1673, ante la acusación de volver a los pecados necesarios, Leibniz dirá: "Respondo que es falso que aquello que se sigue de lo que es necesario sea necesario" (Leibniz, A VI, iii, 127). Y más tarde, en 1677, cuando finalmente adopte la distinción entre necesidad absoluta y necesidad hipotética, Leibniz concluirá que todo lo existente es contingente aunque sea consecuencia de una cosa necesaria, como es la existencia de Dios o la armonía de las $\operatorname{cosas}^{28}$.

Hay otra razón importante para dudar de la identificación de la necesidad de la carta con la necesidad absoluta. Si bien es cierto que la necesidad aludida en el Von der Allmacht und Allwissenheit Gottes und der Freiheit des Menschen parece ser, sin más, la necesidad per se, la posición de Leibniz en dicho escrito es precisamente la contraria: los eventos existentes (y la salvación o condenación derivada de ellos) no son necesarios, la previsión divina no los vuelve necesarios. En el parágrafo 11, Leibniz deja en claro que podemos imaginar o concebir la no realización de aquello que la presciencia divina ha previsto que sucederá ${ }^{29}$. Por tanto, solo puede afirmarse que, $s i$ ha sido previsto por Dios que un evento acontecerá, entonces acontecerá. Esto parece más bien compatible con una necesidad hipotética. Leibniz llega incluso a afirmar que la distinción entre necesidad absoluta y necesidad hipotética constituye el "cabal fundamento" para refutar el sofisma que asocia la predestinación con la necesidad ${ }^{30}$. ¿Por qué, entonces, Leibniz sostendría dos cosas tan contradictorias en dos escritos tan cercanos en el tiempo?

28 Cf. A VI, iii, 129, agregado por Leibniz al manuscrito de la Confessio philosophi en 1677.

29 Cf. A VI, i, 540.

30 Cf. Roldán 65/A VI, i, 541. 


\section{La necesidad de lo mejor: una necesidad sin cualificación}

Muchas son las dificultades que este análisis plantea. No deja de llamar la atención que Leibniz reconozca, ya hacia 1670-1671, la importancia de la distinción tradicional entre las dos clases de necesidad, y que, a pesar de ello, no la incorpore en su filosofía escrita hasta las notas de su diálogo con Stensen en 1677.

Esta demora tal vez pueda entenderse si se tiene en cuenta que el Leibniz de principios de la década de 1670 parece reacio a las disquisiciones y conceptualizaciones escolásticas, sobre todo en lo referido a la cuestión de la predestinación. Como lo afirma en el parágrafo 5 de Von der Allmacht und Allwissensheit Gottes und der Freiheit des Menschen, los "subterfugios escolásticos" y las cuestiones que estos han suscitado "lo han confundido todo" 31 .

Esto permite inferir, a mi juicio, que el Leibniz de la carta a Wedderkopf (ciertamente, más que el de Von der Allmacht...) pretendía deliberadamente evitar toda cualificación respecto de la noción de necesidad que estaba empleando, o que al menos se sentía cómodo con tal actitud. En efecto, si hubiese querido aclarar o precisar a qué tipo de noción modal (escolástica) estaba aludiendo, contaba con suficiente conocimiento como para hacerlo. Asimismo, si bien es cierto que Leibniz conocía las distinciones modales del legado escolástico, sus escritos hasta 1671 revelan una escasa teorización sobre las nociones modales ${ }^{32}$. Esta situación vira drásticamente a partir de la Confessio philosophi de 1672-1673, escrito en el que Leibniz afronta la tarea de precisar cuál es el rasgo definitorio de las modalidades metafísicas.

En este sentido, me parece más seguro interpretar que Leibniz emplea, en su esquema argumental de la carta, una noción modal imprecisa, menos técnica que las que habría de emplear a partir de la Confessio philosophi. Una noción que no se ajusta a la significación de la tradicional necessitas absoluta, en tanto entraña una necesidad derivada de una condición. Aun así, y a pesar de su imprecisión, se trata de una noción que afirma en definitiva la necesidad, y ello parece excluir la contingencia. Prueba de ello es que Leibniz indicó, por escrito, que la posición expresada en la carta debió ser corregida. Y en 1689 señaló, nuevamente, cuán cerca estuvo en su juventud de la admisión de una necesidad absoluta de todas las cosas, aun conjugando esa necesidad con la libertad. Eso es lo que hace el cuarto argumento del esquema. el concepto de "Necessitas".

32 Los escritos jurídicos reunidos en los Elementa juris naturalis muestran, ciertamente, un intento de caracterizar las nociones modales. Pero tal esfuerzo está más orientado a la formulación de ciertos teoremas jurídicos, construidos en base a la analogía entre las modalidades metafísicas y las jurídicas o deónticas (justo, debido, ilícito, indiferente). No hay, en estos pasajes, un análisis profundo del significado de los conceptos modales, como sí se esbozará en la Confessio philosophi. Cf. A VI, i, 466-481; Esquisabel 2011, pp. 10-11. 
Sin embargo, a pesar de expresar una necesidad carente de toda cualificación precisa, la noción modal de la carta no me parece una noción vacía de significación. Si la interpretación aquí propuesta es correcta, tal noción expresa, en definitiva, la necesidad que adquiere lo óptimo en el bosquejo metafísico que Leibniz propone ya en 1671. Esta necesidad tiene su origen en la naturaleza de Dios, sede de la harmonia rerum, y desde esta fuente se transmite a todo lo existente, constituyendo el principio que aporta el único criterio que guía la acción divina. Tal criterio es el de "la armonía más perfecta" o "la belleza de las cosas". Esta necesidad de lo mejor tiene una significación metafísica, en la medida en que se funda en la naturaleza de las cosas, o más precisamente, en la naturaleza de Dios, que por ser una mente perfectísima solo podría ser "necesitado hacia lo óptimo por la misma idealidad de las cosas". Ahora bien, esta necesidad presente en las cosas tiene, para Leibniz, importantes consecuencias para la moral y para la ciencia civil $^{33}$, ya que ella conduce a Dios a buscar el mayor bien para las criaturas racionales, a través del primado de la justicia en la república universal. Así lo expresa en una carta a Hermann Conring de febrero de 1671:

Me parece haber demostrado que hay una cierta razón última de las cosas (es decir, Dios), la armonía universal, la mente más sabia y más poderosa; que las cosas óptimas, las cosas más armónicas ${ }^{34}$, son para ella las cosas más gratas, y por tanto, que lo justo, es decir, lo que es grato a Dios, es todo aquello que es lo más concordante para la armonía de las cosas, para el bien del universo, para la república universal, por así llamarla; y por ende, que la doctrina de derecho natural es la misma que la de la óptima república universal [...] (Leibniz, A II, i, 79).

A mi juicio, esta necesidad de lo mejor que se transfiere a todo lo existente tiene para Leibniz, ya en 1671, un pilar fundamental, que no es otro que su concepción de la naturaleza de la voluntad y su posición intelectualista en torno a la libertad. Ya en la carta a Wedderkopf, Leibniz dedica las líneas finales a argumentar que no hay indiferencia de equilibrio, no hay elección sobre qué se ha de querer, sino que la voluntad quiere indefectiblemente lo mejor. Una voluntad que no tuviese al bien por objeto sería algo monstruoso ${ }^{35}$. El mismo rechazo a una plena indiferencia se encuentra en Von der Allmacht ... ${ }^{36}$, donde Leibniz deja en claro que la tendencia del querer divino hacia lo óptimo sí constituye algo absolutamente necesario, algo cuyo opuesto es contradictorio:

[...] quien es omnisciente quiere aquello que considera óptimo, puesto que resulta consustancial a la sabiduría el querer lo óptimo y quien niegue esto está trastocando los términos. Pues, si se es bueno, se quiere (cuando se comprende su naturaleza) lo mejor -tal y como dice Aristóteles-, si es que lo reconoce. Y, como quien es omnisciente conoce lo mejor, de ahí se sigue que lo quiere (Leibniz, Roldán 70 / A VI, i, 543-544).

33 Cf. A VI, ii, 268.

34 En griego en el original.

35 Cf. A II, i, 118.

36 Cf. A VI, i, 544-546. 
Hay, para el Leibniz de 1671, una conexión necesaria entre la sabiduría y la tendencia de la voluntad hacia lo óptimo, tanto como la hay entre el sabio y la búsqueda de armonía. Todo sabio, dice Leibniz, "es deleitado por la belleza o armonía” (Leibniz, A VI, i, 434$435)^{37}$. Esta concepción de la voluntad y de la conexión entre la naturaleza de Dios y la mayor armonía es la que subyace, según creo, a la necesidad que se transfiere a los eventos del mundo actual. Todas las cosas son necesarias porque, en última instancia, su existencia depende de un ser que está necesitado a querer y elegir lo mejor.

\section{Consideraciones finales, un necesitarismo teísta}

La necesidad que Leibniz atribuye a la cadena de eventos existentes en la carta a Wedderkopf de 1671 no puede identificarse, a mi juicio, con la necessitas absoluta que el filósofo de Hannover tomaría del legado escolástico, años más tarde, para su propia metafísica. Ello se debe a que la modalidad invocada en la misiva admite el principio modal expresado por el Axioma de distribución, esto es, se distribuye sin problemas entre el antecedente y el consecuente de un condicional. Pero el Leibniz de la Confessio philosophi no habría de aceptar este principio de distribución. Además, el Leibniz de 1671 no admitiría una consecuencia fundamental de la necesidad absoluta de las cosas creadas, como sería el carácter contradictorio (por ende imposible) de los otros mundos posibles no actualizados. En la misma carta, Leibniz afirma que Dios elige estas cosas a partir de un infinito número de cosas posibles. Por lo demás, Leibniz parece querer evadir toda cualificación de la necesidad atribuida a la serie de eventos, y manifiesta en la época una profunda desconfianza en las distinciones conceptuales escolásticas.

La necesidad que Leibniz atribuye a todo lo existente no parece ser otra que la necesidad (ciertamente metafisica) con la cual Dios, en tanto mente supremamente sabia, es compelido a querer y elegir lo óptimo, tomando como único criterio de acción la armonía más perfecta o la belleza de las cosas. Esta necesidad se transfiere a su consecuencia irremediable, la existencia de lo más perfecto. Tal modalidad parece adecuarse más, en definitiva, a la tradicional necesidad de la consecuencia, la necesidad con la cual un consecuente se sigue de una condición. Sin embargo, el Leibniz posterior vio en este esquema argumental el riesgo del "precipicio" de la necesidad absoluta ${ }^{38}$. Ello sugiere que la conclusión modal de la carta iba un poco más lejos que la atribución de una mera necesidad hipotética.

La forma de necesitarismo a la que Leibniz arribó en esta carta bien podría calificarse como un necesitarismo teísta, puesto que pone a un Dios personal como fundamento ontológico o primer principio de todas las cosas. Según tal concepción, todo lo actual existe necesariamente (con una necesidad simpliciter, sin cualificación), porque tiene su razón última en un ser necesario (en tanto ratio plena) cuya suprema 
libertad consiste en ser conducido necesariamente (ser necesitado) hacia lo más perfecto. En otras palabras, se trataría de un necesitarismo en el cual la naturaleza de la ultima ratio rerum vuelve necesaria la existencia del mundo óptimo.

El Leibniz de los años posteriores a la carta a Wedderkopf introducirá mayores precisiones en la formulación de su metafísica modal. La necesidad de las cosas existentes será la necesidad hipotética, una necesidad compatible con la contingencia. La idea misma del necesitarismo será reinterpretada en términos de la atribución de una necesidad absoluta a las cosas existentes. Tal concepción será rechazada sobre la base del reconocimiento de los "posibles que ni son, ni serán, ni fueron" 39 . Sin embargo, la concepción subyacente al esquema de 1671 persistirá, cabe notarlo, a través de lo que el Leibniz de 1677 denominará como la necessitas eligendi optimum ${ }^{40}$, la necesidad con la cual Dios elige siempre lo mejor. Sobre este principio y su estatuto modal, Leibniz tendrá mucho que reflexionar a lo largo de su vida. Pero es claro que a través de esta verdad persistente en su obra, referida a la elección divina de lo mejor, el Leibniz de los últimos años consagrará un principio de enorme valor en su física y su metafísica de madurez: el principio de lo mejor.

\section{Referencias bibliográficas}

\section{FUENTES Y ABREVIATURAS}

Ediciones de los escritos de Leibniz

A: Leibniz, G. W. Sämtliche Schriften und Briefe, Herausgegeben von der BerlinBranderburgischen Akademie der Wissenschaften und der Akademie der Wissenschaften in Göttingen. Darmstadt 1923 y ss., Leipzig 1938 y ss., Berlin 1950 y ss., Münster 1999 y ss.

G: $\quad$ G. W. Leibniz. Die philosophischen Schriften, ed. C. I. Gerhardt, 7 vols. Berlin: Weidman 1875-1890; reimpresiones Hildesheim: Georg Olms 1960-61 y Hildesheim-New York: Georg Olms 1978.

Gr: Grua, G. (ed.) (1948), G. W. Leibniz. Testes inédits d'après les manuscrits de la bibliothèque provinciale de Hanovre. Paris: Presses Universitaires.

Traducciones al inglés

Sl: $\quad$ Sleigh, Robert C. Jr. (2001), Papers Concerning the Problem of Evil, 16711678. New Haven \& Londres: Yale University Press.

39 Cf. A VI, iv, 1653.

40 Cf. A VI, iv, 1351-1352. 
Traducciones al castellano

de Olaso: Ezequiel de Olaso (comp.) (1982), G. W. Leibniz. Escritos filosóficos. Buenos Aires: Charcas.

Roldán: Roldán Panadero, Concha (comp.) (1990), Gottfried Wilhelm Leibniz. Escritos en torno a la libertad, el azar y el destino. Madrid: Tecnos.

Otras fuentes y obras consultadas

Stahl: Daniel Stahl, Compendium metaphysicae in XXIV tabellas redactum (Francofurtum 1686). Münchener Digitalisierungs Zentrum, Digital Bibliothek. Permalink: http://www.mdz-nbn-resolving.de/urn/resolver.pl?urn=urn:nbn: de:bvb:12-bsb10943995-0.

Tomás de Aquino, Corpus Thomisticum. S. Thomae de Aquino Opera Omnia, recognovit ac instruxit Enrique Alarcón automato electronico. Pompaelone ad Universitatis Studiorum Navarrensis, 2013. http://www.corpusthomisticum. org/iopera.html

Los textos se citarán indicando la edición, el tomo (si correspondiere) y el número de página. Los textos de Leibniz correspondientes a las ediciones en castellano se citarán indicando también la ubicación consignada por el traductor (cuando la referencia exista o haya sido chequeada). Donde no esté indicado, las traducciones son mías.

\section{BiBLIOGRAFÍA SECUNDARIA}

Adams, R. M., (1994), Leibniz. Determinist, Theist, Idealist. Oxford: Oxford University Press.

Anfray, J. P. (2011), "Leibniz, le choix du meilleur et la nécessité morale", en P. Rateau, ed., Lectures et interprétations des Essais de théodicée de G. W. Leibniz, Studia Leibnitiana - Sonderhefte 40. Stuttgart: Franz Steiner Verlag, pp. 59-78.

Antognazza, María Rosa (1999), "Immeatio and Emperichoresis. The Teological Roots of Harmony in Bisterfeld and Leibniz", en S. Brown, ed., The Young Leibniz and his Philosophy (1646-76). Dordrecht: Kluwer Academic Publishers, pp. 41-64.

Begby, E. (2005), "Leibniz on Determinism and Divine Foreknowledge", Studia Leibnitiana 37: 83-98.

Broadie, Alexander (1999), Introduction to Medieval Logic. Oxford: Oxford University Press.

Carraud, Vincent (2002), Causasive ratio. La raison de la cause, de Suarez à Leibniz. Paris: PressesUniversitaires de France.

Couturat, Louis (1961), La logique de Leibniz. Reimpresión: Hildesheim: Georg Olms.

(1972), “On Leibniz`s Metaphysics”, en Frankfurt (1972: 19-45). 
Curley, Edwin M., 1972, “The Root of Contingency”, en H. Frankfurt, ed., Leibniz: A Collection of Critical Essays. Garden City, New York: Doubleday Anchor, pp. 67-97.

Davidson, J. D. (2005), "Video meliora proboque, deteriora sequor. Leibniz on the Intellectual Source of Sin", en D. Rutherfordy J. A. Cover, eds., Leibniz.Nature and Freedom. Oxford: Oxford University Press, pp. 234-253.

Dascal, M. (ed.) (2008), Leibniz: What Kind of Rationalist? Tel Aviv: Springer.

Echevarría, Agustín (2011), Metafisica leibniziana de la permisión del mal. Navarra: Eunsa.

Escobar Viré, Maximiliano (2011), "El argumento ontológico, la necesidad absoluta y el problema de la contingencia en Leibniz", Revista Latinoamericana de Filosofía, Vol. XXXVII (1): 97-125.

"La metafísica modal de Leibniz: su fundamentación de la contingencia hacia 1686 y su concepción integral de madurez", Diánoia Vol. LIX, número 73 (noviembre de 2014): 47-72.

"La necesidad moral en Leibniz: su contenido alético y su significación específica", Revista Latinoamericana de Filosofía Vol. XL, No 2 (primavera de 2014): 145-170.

Esquisabel, Oscar (2011), "El tratamiento de las modalidades aléticas y deónticas en Leibniz", en J. Legris, comp., Documentos del CIECE 7. Perspectivas en Lógica Deóntica. Buenos Aires: Centro de Investigación en Epistemología de las Ciencias Económicas, pp. 7-23.

Friedman, Russell L. y L. O. Nielsen (eds.) (2003), The Medieval Heritage in Early Modern Metaphysics and Modal Theory, 1400-1700. Dordrecht: Springer.

Garson, James W. (2006), Modal Logic for Philosophers. Cambridge: Cambridge University Press.

(2009), "Modal Logic", The Stanford Encyclopedia of Philosophy (Winter 2009 Edition), Edward N. Zalta (ed.), URL = <http://plato.stanford. edu/archives/win2009/entries/logic-modal/>.

Greenberg, S. (2005), "Leibniz Against Molinism: Freedom, Indifference and the Nature of the Will", en D. Rutherfordy J. A. Cover, eds., Leibniz. Nature and Freedom. Oxford: Oxford University Press, pp. 217-233.

Grua, Gaston (1953), Jurisprudence universelle et Théodicée selon Leibniz. Paris: Presses Universitaires de France.

Jolley, Nicholas (ed.) (1995), The Cambridge Companion to Leibniz. Cambridge: Cambridge University Press.

Knebel, Sven (2003), "The Renaissance of Statistical Modalities in Early Modern Scholasticism", en Friedman, Russell L. y Nielsen, L. O., eds., The Medieval Heritage in Early Modern Metaphysics and Modal Theory, 1400-1700. Dordrecht: Springer, pp. 231-251. 
Knuuttila, Simo (1988), Modern Modalities. Studies of the History of Modal Theories from Medieval Nominalism to Logical Positivism. Dordrecht-Boston-Londres: Kluwer Academic Publishers.

(2008), "Medieval Modal Theories and Modal Logic", en D. Gabbay y J. Woods, comps., Handbook of the History of Logic vol. 2, Mediaeval and Renaissance Logic. Amsterdam: Elsevier, pp. 505-578.

Kremer, Elmar J. y Michael J. Latzer, eds. (2001), The Problem of Evil in Early Modern Philosophy. Toronto: University of Toronto Press.

Laerke, Mogens (2008), Leibniz lecteur de Spinoza. La genèse d'une opposition complexe. Paris: Honoré Champion Éditeur.

Moll, Konrad (1999), "Deus sive harmonia universalis est ultima ratio rerum: the conception of God in Leibniz's early philosophy", en S. Brown, ed., The Young Leibniz and his Philosophy (1646-76). Dordrecht: Kluwer Academic Publishers, pp. 65-78.

Murray, M. J. (1995), "Leibniz on Divine Foreknowledge of Future Contingents and Human Freedom", Philosophy and Phenomenological Research 55: 75-108.

(1996), "Intellect, Will and Freedom: Leibniz and his Precursors", Leibniz Review 6: 25-59.

(2005), "Spontaneity and Freedom in Leibniz", en D. Rutherford y J. A. Cover, eds., Leibniz. Nature and Freedom. Oxford: Oxford University Press, pp. 194-216.

Piro, F. (1999), "Leibniz and ethics: the years 1669-72", en S. Brown, ed., The Young Leibniz and his Philosophy (1646-76). Dordrecht: Kluwer Academic Publishers, pp. 147-167.

(2008), "For a History of Leibniz's Principle of Sufficient Reason. First Formulations and their Historical Background", en M. Dascal, ed., Leibniz: What Kind of Rationalist? Tel Aviv: Springer, pp. 463-478.

Poser, H. (1969), Zur Theorie der Modalbegriffe bei G. W. Leibniz, Studia Leibnitiana Supplementa VI. Wiesbaden: Franz Steiner Vg.

Rateau, Paul (2008), La question du mal chez Leibniz. Fondaments et élaboration de la Théodicée. Paris: Honoré Champions Éditeur.

Rescher, N. (1952), "Contingence in the Philosophy of Leibniz", The Philosophical Review 61: 26-39.

(1967), The Philosophy of Leibniz. Englewood Cliffs: Prentice-Hall.

(1981), Leibniz's Metaphysics of Nature. Dordrecht: D. Reidel Publishing Company.

(2001), "Contingentia Mundi. Leibniz on the World's Contingency", Studia Leibnitiana 33: 145-162.

(2002), "Leibniz on God's Free Will and the World's Contingency", Studia Leibnitiana 34: 208-220.

Rutherford, D. (1995), Leibniz and the Rational Order of Nature. Cambridge: Cambridge University Press. 
Rutherford, D. y J. A. Cover (eds.) (2005), Leibniz. Nature and Freedom. Oxford: Oxford University Press.

Schepers, H. (1999), "Posibilidad y contingencia. Historia de la terminología filosófica anterior a Leibniz", Revista de Filosofía y Teoría Política 33: 43-63.

Sleigh, Robert C. (1990), Leibniz \& Arnauld. A Commentary on Their Correspondence. New Haven/London: Yale University Press.

(1982), "Truth and Sufficient Reason in the Philosophy of Leibniz", en M. Hooker, ed., Leibniz: Critical and Interpretive Essays. Minneapolis: University of Minnesota Press, pp.209-242. 